\title{
Downregulation of Dicer enhances tumor cell proliferation and invasion
}

\author{
LEI HAN ${ }^{1,2^{*}}$, ANLING ZHANG ${ }^{1,2^{*}}$, XUAN ZHOU $^{1,2,3}$, PENG XU $^{1}$, GUANG-XIU WANG $^{1}$, \\ PEI-YU PU ${ }^{1,2}$ and CHUN-SHENG KANG ${ }^{1,2,4}$
}

\begin{abstract}
${ }^{1}$ Laboratory of Neuro-Oncology, Tianjin Neurological Institute; ${ }^{2}$ Department of Neurosurgery, Tianjin Medical University General Hospital, Tianjin 300052; ${ }^{3}$ Department of First Head and Neck Cancer, Tianjin Medical University Cancer Institute and Hospital, Tianjin 300060; ${ }^{4}$ Tianjin Key Laboratory of Nerve Injury, Variation and Regeneration, Tianjin 300052, P.R. China
\end{abstract}

Received January 19, 2010; Accepted March 26, 2010

DOI: 10.3892/ijo_00000678

\begin{abstract}
RNAs are non-coding, single-stranded RNAs that regulate target gene expression by repressing translation or promoting RNA cleavage. Dicer is an essential component of the miRNA processing machinery. To identify a role for miRNAs in tumorigenesis, we designed an adenovirus expressing small hairpin RNA (shRNA) to silence Dicer and globally suppress the maturation of miRNAs. We identified that the impairment of miRNA processing conferred an enhanced proliferative activity and invasive ability on each of three tumor cell lines in vitro. Inhibition of Dicer was associated with activation of p-AKT and enhanced expression of the cell cycle associating molecules, cyclin A and PCNA, as well as MMP-2 and MMP-9, proteins involved in tumor cell invasion. Adenoviral gene silencing of Dicer in subcutaneous MCF-7 xenografts significantly increased tumor growth in vivo compared to tumors infected with non-loading adenovirus. Increased tumor growth was associated with p-AKT activation and upregulation of cyclin A, PCNA MMP-2 and MMP-9. These findings demonstrate that global reduction of miRNA processing by silencing Dicer enhances tumor proliferation and invasion, and the p-AKT pathway may contribute to this phenotype via the downstream molecules, cyclin A, PCNA, MMP-2 and MMP-9.
\end{abstract}

\section{Introduction}

Since the discovery in C. elegans of the first miRNA, lin-4, thousands of miRNAs have been identified across diverse species ranging from humans to viruses, suggesting critical

Correspondence to: Dr Chunsheng Kang, Laboratory of NeuroOncology, Tianjin Neurological Institute, 152 Ahshan Road, Heping, Tianjin 300052, P.R. China

E-mail: kang97061@yahoo.com

*Contributed equally

Key words: cancer, Dicer, AKT, proliferation, invasion and potentially broad biological roles for miRNAs. Multiple reports indicate that miRNAs in some systems regulate development, cell proliferation, apoptosis and other important physiological processes (1). As miRNAs play a prominent role in the regulation of many basic biological events, much interest has focused on the contribution of miRNAs in cancer progression. Recent studies have shown that the miRNA expression profile is altered in various tumors (2-9). Of note, Lu et al observed a general downregulation of miRNAs in tumors compared with normal tissue (2). Moreover, the change of miRNA expression in tumors was associated with the developmental lineage and differentiation state of the tumors.

miRNA biogenesis requires a set of proteins collectively referred to as the miRNA machinery. This machinery contains Dicer, an enzyme that belongs to the RNase III endonuclease family, and is required for processing of long double-stranded RNA (dsRNA) or microRNA (miRNA) precursors into mature effector RNA molecules (10). miRNAs encoded in the genome are transcribed by RNA polymerase II into primary transcripts, called pri-miRNAs. The pri-miRNA is cleaved to a 6070-nt stem-loop, termed a miRNA precursor (pre-miRNA), by the RNase III endonuclease Drosha. Pre-miRNAs are actively transported from the nucleus to the cytoplasm by Exportin 5 (11), where Dicer cuts both strands of the premiRNA duplex, generating a mature 21-nucleotide miRNA duplex $(12,13)$. The 21-nucleotide miRNA duplex is then incorporated into a ribonucleoprotein complex, called the RNA-induced silencing complex (RISC), to repress translation. As Dicer is essential for the processing of miRNAs, downregulation of Dicer globally impairs miRNA processing and activity. Investigation of the biological function of Dicer revealed its essential role in cell development and differentiation in several species (14-16).

The role of Dicer and miRNA in cell growth and differentiation implies that these molecules, in an unknown manner, may impact tumorigenesis. Many studies focusing on Dicer have confirmed this correlation, and revealed that Dicer plays either a negative or positive role in tumor transformation in different types of cancers $(3,17,18)$. To address the broad role for miRNAs in tumorigenesis, we designed an adenovirus to generate shRNA and silence Dicer expression, thereby impairing miRNA processing. Dicer downregulation increased the proliferative and invasive ability of tumor cells in vitro, 
and increased expression of a set of proteins associate with these phenotypes. Dicer downregulation similarly increased subcutaneous tumor xenograft proliferation in vivo. Taken together, our data demonstrate that Dicer inhibition dramatically increases the malignant transformation of cancer cells in vitro and tumor growth in vivo.

\section{Materials and methods}

shRNA sequence and the construction of adenovirus. A recombinant adenovirus encoding shRNAs against Dicer (Ad-Dicer) was synthesized by Wuhan Genesil Co., Ltd. The virus carried three sequences, each of which targeted different sites of the Dicer genes. The sequences of the shRNAs were as follows: site A, sense 5'-TGC TTG AAG CAG CTC TGG Adtdt-3', anti-sense 5'-TCC AGA GCT GCT TCA AGC Adtdt-3'; site B, sense 5'-GGA CGG TGT TCT TGG TCA Adtdt-3', antisense 5'-TTG ACC AAG AAC ACC GTC Cdtdt-3'; site C, sense 5'-AAA GAG CTG TCC TAT CAG ATC dtdt-3', antisense 5'-GAT CTG ATA GGA CAG CTC TTT dtdt-3'. The non-load adenovirus (Ad-HK) was used as a negative control.

Cell culture and adenovirus infections. Human cancer cell lines U251, MCF-7 and SCG7901 were obtained from the Institute of Biochemistry and Cell Biology, Chinese Academy of Sciences. Cells were cultured in DMEM medium supplemented with $10 \%$ fetal bovine serum (FBS). All cultures were maintained at $37^{\circ} \mathrm{C}$ in a moist atmosphere containing $5 \% \mathrm{CO}_{2}$. For infection, trysinized cells were plated in 6-well plates at $2 \times 10^{5}$ cells per well. Infection with Ad-Dicer (MOI=100) was performed in serum-free medium on cells reaching 50-80\% confluence. After 4 h of infection, the cells were restored to complete medium.

RT-PCR analysis. Total RNA was extracted with TRIzol (Gibco, USA) $48 \mathrm{~h}$ after infection, and reverse transription was performed using AMV reverse transcriptase (Takara, Japan) according to the manufacturer's protocol. Dicer was amplified under the reactive conditions: $94^{\circ} \mathrm{C}, 5 \mathrm{~min} ; 94^{\circ} \mathrm{C}$ $30 \mathrm{sec}, 52^{\circ} \mathrm{C} 30 \mathrm{sec}, 72^{\circ} \mathrm{C} 30 \mathrm{sec}, 40$ cycles; $72^{\circ} \mathrm{C}, 5 \mathrm{~min}$. $\beta$-actin was used as control. Oligonucleotide sequences used to amplify Dicer were: forward 5'-CGA TAA CTT TAT TGG AGA TTT AC-3', reverse 5'-ATT GGG TGT CCC GAA GAG TT-3'; for $\beta$-actin: forward 5'-TCC CTG GAG AAG AGC TAC GA-3', reverse 5'-GAT CCA CAC GGA GTA CTT GC-3'.

Transwell cell migration assay. The top chamber of a transwell chamber was incubated with $60 \mu 1$ Matrigel diluted with DMEM (1:2, Matrigel: DMEM) at $37^{\circ} \mathrm{C}$ for $30 \mathrm{~min}$, and allowed to polymerize. Infected cells were trypsinized, adjusted to $1 \times 10^{6} / \mathrm{ml}$ in DMEM, and $200 \mu \mathrm{l}$ of the resuspended cell solution was added to the top chamber above the Matrigel. The bottom chamber was filled with $600 \mu 1$ of chemoattractant solution. The transwell plate was assembled and incubated at $37^{\circ} \mathrm{C}$, in a $5 \% \mathrm{CO}_{2}$ incubator. After $24 \mathrm{~h}$, the top chamber was removed, and the Matrigel and unmigrated cells were gently scraped with a wet cotton swab. Cells were stained by hematoxylin for $5 \mathrm{~min}$, and washed with PBS to remove excess stain. Finally, the membrane was removed from the chamber with the migrated side placed down onto a glass slide, administered a few drops of Permount, and covered with a cover slide. The average number of migrated cells per field was quantified under high power (x200).

Flow cytometry. Forty-eight hours after infection, adherent cells were trypsinized, placed in suspension, and washed with PBS. Cells were fixed in $75 \%$ ethanol at $4^{\circ} \mathrm{C}$ overnight. Cells were then washed twice with $\mathrm{PBS}$, and incubated with $200 \mu 1 \mathrm{RNase} A(1 \mathrm{mg} / \mathrm{ml})$ at $37^{\circ} \mathrm{C}$ for $30 \mathrm{~min}$. Cells were then stained in the dark with $800 \mu 1$ propidium iodide staining solution for $30 \mathrm{~min}$ at $4^{\circ} \mathrm{C}$. Analysis was performed on a FACSCalibur flow cytometer (Bio-Rad, USA).

MTT assay. Cells were plated in a 96-well plate at a density of 2000 cells per well $48 \mathrm{~h}$ after infection. Cells were incubated with $20 \mu \mathrm{l}$ MTT solution $(5 \mathrm{mg} / \mathrm{ml})$ 24, 48, 72, 96, 120,144 and $168 \mathrm{~h}$ following infection at $37^{\circ} \mathrm{C}$ for $4 \mathrm{~h}$. At the end of the incubation period, the solution was aspirated, and $200 \mu \mathrm{l}$ dimethyl sulfoxide were added to each well. The absorbance was measured at a wavelength of $570 \mathrm{~nm}$ with background subtraction at $650 \mathrm{~nm}$. Mean was determined for 6 replicate wells. Cell viability was defined as the absorbance of treated/the control.

Western blot analysis. Total proteins were extracted and the protein concentration was determined by Lowry method $48 \mathrm{~h}$ following infection. Protein lysates ( $40 \mu \mathrm{g}$ ) from each sample were subjected to SDS-PAGE separation, and transferred to a PVDF membrane. The membrane was incubated with primary antibodies against Dicer, p-AKT, MMP2, MMP9, cyclin A and PCNA (Santa Cruz; 1:500 dilution), followed by HRP-conjugated secondary protein (Zymed, San Diego, CA; 1:1000 dilution). The specific protein was detected using a SuperSignal protein detection kit (Pierce, USA). After washing with stripping buffer, the membrane was reprobed with antibody against $\beta$-actin (1:500 dilutions). The band density of specific proteins was quantified after normalization with the density of $\beta$-actin.

Subcutaneous tumor model and gene therapy in vivo. All experimental procedures were carried out according to the regulations and internal biosafety and bioethics guidelines of Tianjin Medical University and the Tianjin Municipal Science and Technology Commission. Female BALB/c-nu mice ( 6 weeks old) were purchased from the animal center of the Cancer Institute of Chinese Academy of Medical Sciences, bred at the Facility of Laboratory Animals, Tianjin University, and housed in microisolator individually ventilated cages with water and food ad libitum.

MCF-7 cells were injected subcutaneously to generate xenografts on both flanks as described (19). Adenoviral infection was performed once tumor size reached approximately $5 \mathrm{~mm}$ in diameter, and again three days thereafter. At this time, one flank was infected with Ad-Dicer by intratumoral injection, while the other was infected with Ad-HK. Tumor volume was measured every 3 days for a 20-day period. At the end of the 20 days, mice were sacrificed, tumors were excised and the tumor specimens were prepared as paraffinembedded sections. The expression levels of Dicer, p-AKT, 
A
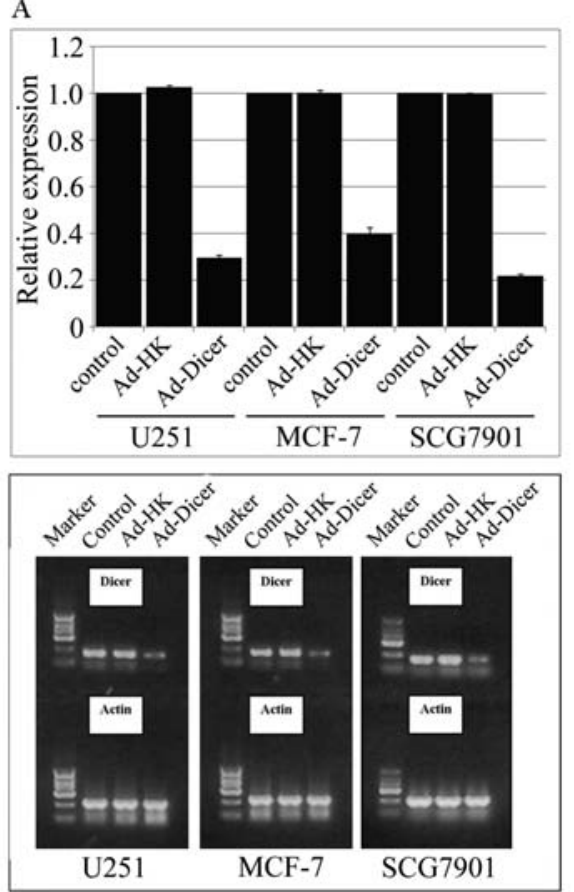

B
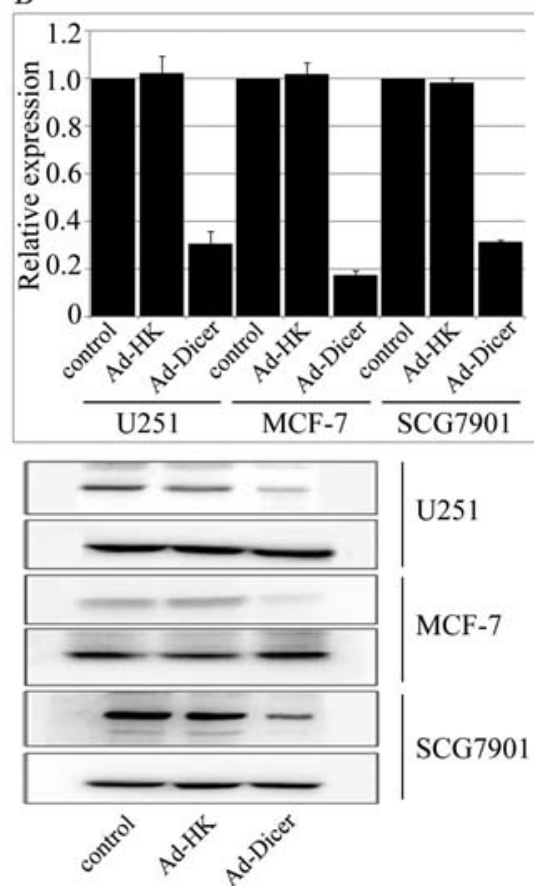

Figure 1. Dicer expression was effectively inhibited by RNA interference mediated by recombinant adenovirus vector. (A) Expression of Dicer mRNA in tumor cell line U251, MCF-7 and SCG7901 was evaluated by RT-PCR assay after transfection. B-actin was used as a negative control. The relative expressions of Dicer mRNA were 29.43, 39.41 and $21.59 \%$ in U251, MCF-7 and SCG7901, respectively. (B) The level of Dicer protein was detected by Western blot. Dicer expression was 30.49, 17.39 and $31.13 \%$ of untreated control in U251, MCF-7 and SCG7901, respectively, as determined dositometry following Western blot assay.

MMP2, MMP9, cyclin A and PCNA were examined by immunohistochemical staining.

Statistical analysis. SPSS16.0 was used for statistical analysis. One-way analysis of variance and Spearman rank correlation analysis were used to analyze the significance between groups. The LSD method of multiple comparisons with parental and control groups was used when the probability for analysis of variance was statistically significant. Statistical significance was determined at $\mathrm{p}<0.05$.

\section{Results}

Inhibition of Dicer expression by adenovirus. As previous studies demonstrated that Dicer is required for dsRNA maturation (20), we assessed the impact of globally impairing miRNA expression on tumor proliferation and invasion by downregulating the expression of Dicer. We constructed a recombinant adenovirus to generate shRNAs targeting Dicer mRNA. Cells infected with Ad-Dicer had significantly lower expression of Dicer mRNA than control and Ad-HK infected groups (Fig. 1A), as determined by RT-PCR. In addition, Western blot analyses demonstrated a significant decrease in the expression of Dicer (Fig. 1B). Thus, the expression of Dicer was effectively inhibited at both the mRNA and protein level by Ad-Dicer infection.

Dicer silencing increased tumor cell malignant phenotype. To measure the impact of Dicer on the invasive ability of tumor cells, we quantified cell migration using the transwell assay. Infection of cells with Ad-Dicer resulted in an increase in the number of cells that migrated through the membrane, when compared to control and Ad-HK infected groups. In U251 cells, infection with Ad-Dicer resulted in $89.33 \pm 1.75$ vs. $55.83 \pm 3.06$ cells and $55.00 \pm 2.83$ cells in control and Ad-HK infected groups, respectively (Fig. 2). Similarly, infection of MCF-7 cells with Ad-Dicer increased migration to $89.00 \pm 2.37$ vs. $58.83 \pm 2.40$ and $58.17 \pm 1.72$ cells in control and Ad-HK, respectively, and infection of SCG7901 cells with Ad-Dicer increased migration to $99.50 \pm 2.07$ cells

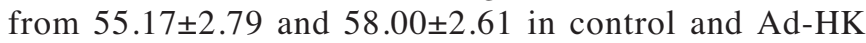
infected groups, respectively (Fig. 2). Therefore, we conclude that knockdown of Dicer significantly enhances cell invasive ability in vitro.

To measure the impact of Dicer on the proliferative capacity of tumor cells, we investigated the impact of rAd-Dicer infection on cell cycle progression, as determined by flow cytometry following PI staining, and cell proliferation, as determined by the MTT assay. Cell cycle analysis revealed that in all 3 cancer cell lines, the rate of S phase strongly increased, accompanied by a reduction in G0/G1 phase and a weak increase in $\mathrm{G} 2 / \mathrm{M}$ phase (Fig. 3A). These data implied that the cell cycle was promoted to $\mathrm{S}$ phase upon knockdown of Dicer. The MTT assay corroborated these findings, demonstrating that Ad-Dicer increased cell proliferation in comparison to the control treated groups (Fig. 3B). Taken together, infection of U251, MCF-7 or SCG7901 cells with Ad-Dicer results in enhanced tumor cell invasive and proliferative capacity compared to control and Ad-HK infected groups. 

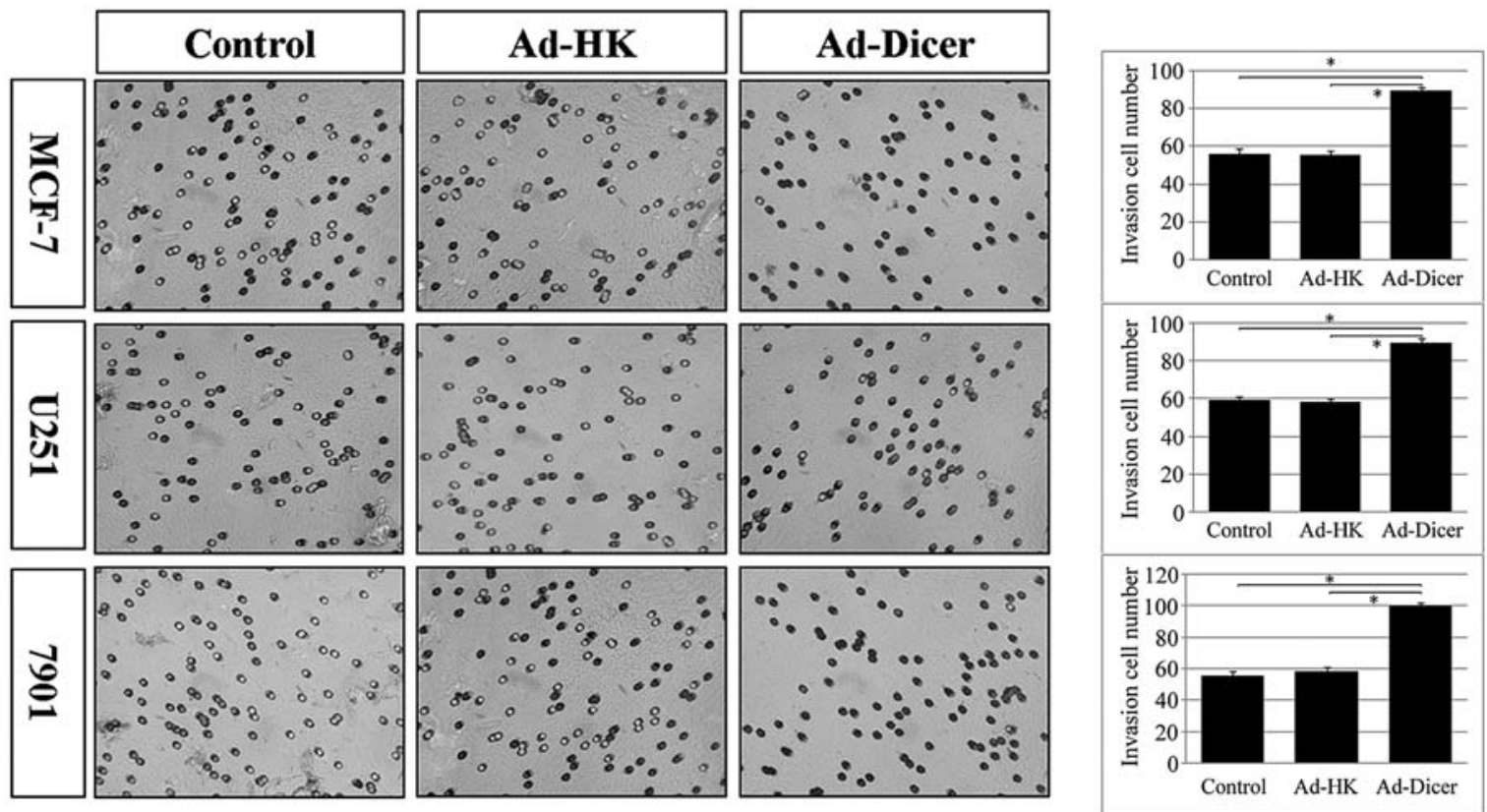

Figure 2. Cell migration ability is increased by infected with Ad-Dicer. Knockdown of Dicer expression increased the ability of MCF-7, U251 and 7901 cells to migrate across a Matrigel membrane, as measured by the transwell assay (x200). The Ad-Dicer group had significantly more cells penetrating membrane than mock or Ad-HK infected groups. Values represent mean \pm SEM ( $\mathrm{n}=5$ for each group). U251: 89.33 $\pm 1.75,55.83 \pm 3.06$ and $55.00 \pm 2.83$ cells in Ad-Dicer, Ad-HK and mock-infected groups, respectively; MCF-7: $89.00 \pm 2.37,58.83 \pm 2.40$ and $58.17 \pm 1.72$ cells, respectively; SCG7901: 99.50 $\pm 2.07,55.17 \pm 2.79$ and $58.00 \pm 2.61$ cells, respectively. ${ }^{*} \mathrm{p}<0.01$.
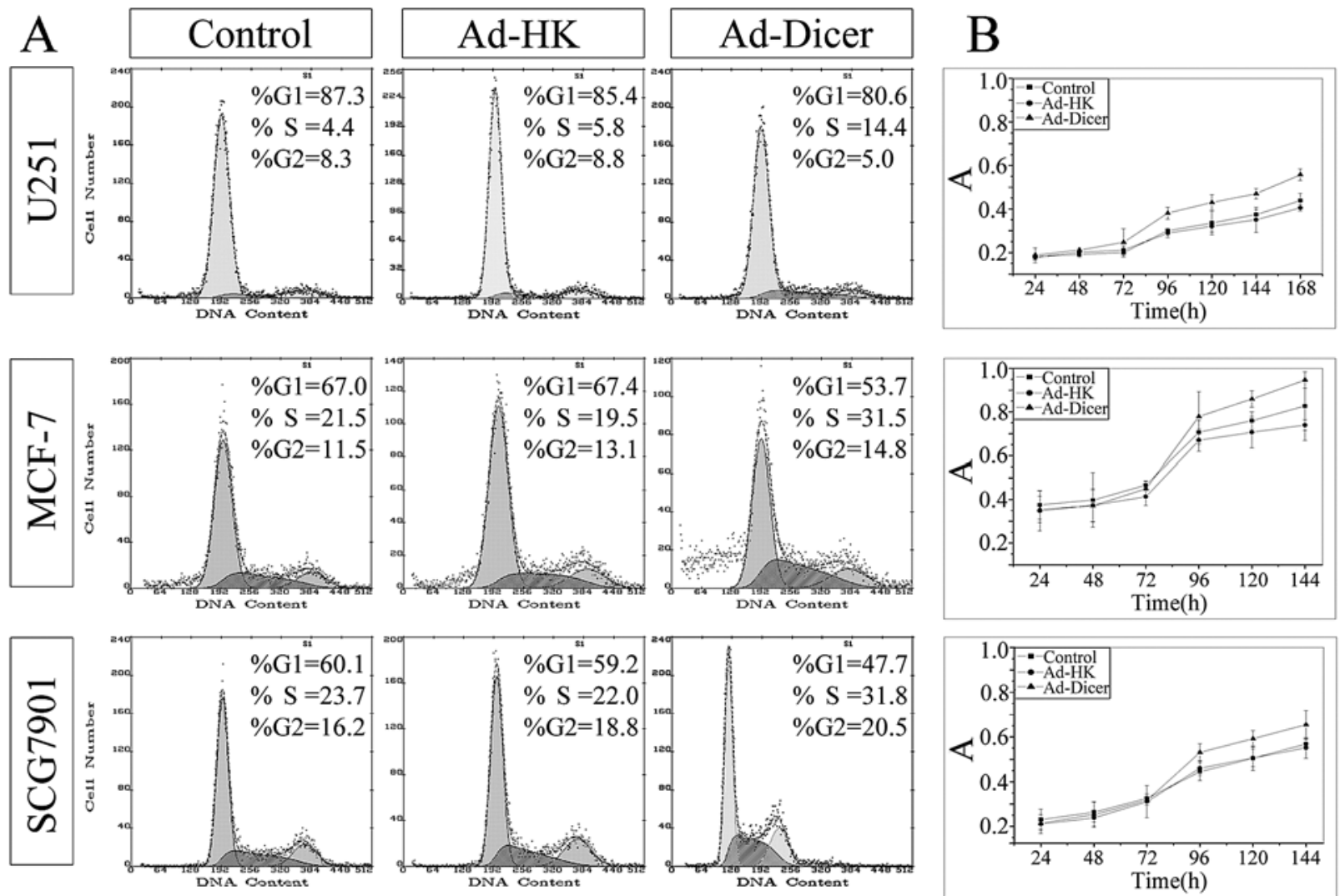

Figure 3. Dicer downregulation increased cell proliferation. Cell viability was detected by cell cycle analysis (A) and MTT assay (B). (A) The cell cycle kinetics of the three groups was detected by flow cytometry and identified a promotion of the cell cycle to S phase by Dicer inhibition in all three cancer cell lines. Cells in S phase represented 14.4, 4.4 and 5.8\% of U251 cells in Ad-Dicer, Ad-HK and mock-infected groups, respectively; $31.5,21.5$ and $19.5 \%$ of MCF-7 cells, respectively; and 31.8, 23.7 and $22.0 \%$ of SCG7901 cells, respectively. (B) MTT assay results corresponded to the cell cycle assay. In all three cancer cell lines, greater number of viable cells, representing an increased proliferative ability, was observed after Ad-Dicer infection compared to Ad-HK or mock-infected groups. 


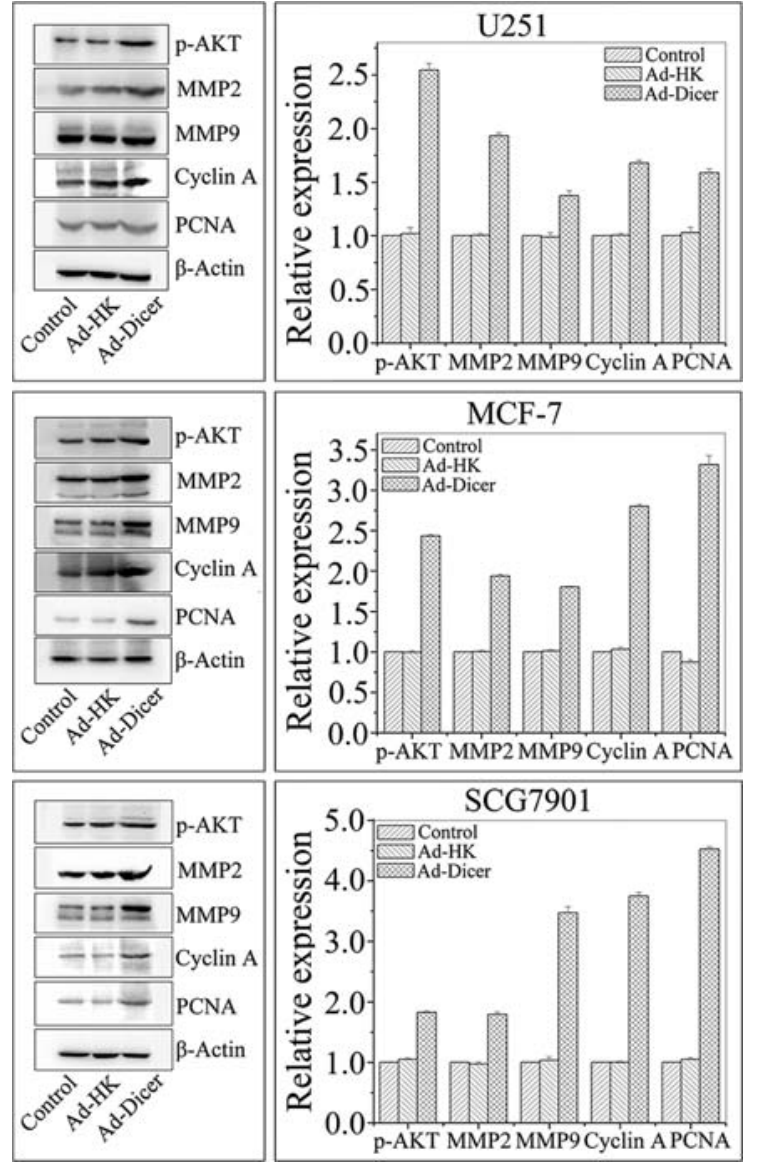

Figure 4. Knockdown of Dicer expression increases expression of proteins downstream of p-AKT pathway. Proteins associated with cell invasive ability and cell cycle was detected by Western blot, including p-AKT, MMP2/9, cyclin A and PCNA. Ad-Dicer infection of U251, MCF-7, SCG7901 cells increased the expression of each of these proteins compared to Ad-HK or mock infection.

Downregulation of Dicer activates $p$-AKT. While we identified a role for Dicer inhibition on tumor cell transformation in vitro, the mechanism contributing to this phenomenon remains unknown. As p-AKT pathway is constitutively activated in a wide range of tumor types, and triggers a cascade of responses that drive tumor progression including cell growth, proliferation, survival and motility, we evaluated the activity of p-AKT in cells in which Dicer was downregulated. Western blot analysis revealed increased phosphorylation of AKT in cells where miRNAs processing was impaired by Dicer downregulation (Fig. 4). To elucidate the mechanism by which downregulation of Dicer impacts cell invasion and proliferation, we assessed the expression of MMP2/9, cyclin A and PCNA. Western blot analysis revealed that the expression of each of these proteins was increased upon downregulation of Dicer (Fig. 4). Therefore, we speculate that the transformation of tumor cells by Dicer downregulation may be a result of the activation of $\mathrm{p}$-AKT and its downstream molecules, MMP2/9, cyclin A, PCNA.

Dicer downregulation initiates tumor transformation in an MCF-7 breast cancer xenograft model. Our in vitro experiments demonstrated that Dicer downregulation could promote the proliferation of tumor cells. To investigate how downre-

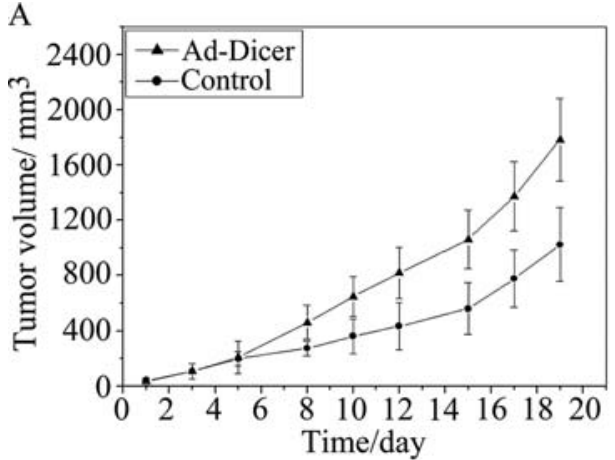

B

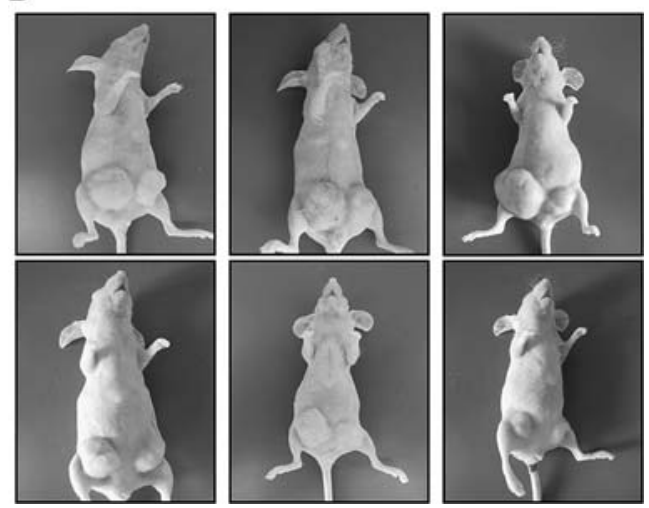

Figure 5. Ad-Dicer infection increases tumor growth in a MCF-7 subcutaneous tumor xenograft model. Xenografts were planted on both sides of the mouse, and Ad-Dicer, or Ad-HK infection was performed by intratumoral injection. The left tumor was treated with recombinant adenovirus that expresses siRNA against Dicer, and the right was treated by non-loading adenovirus. (A) Significant differences between the Ad-Dicer and Ad-HK infected tumors were observed. (B) Tumors infected with Ad-Dicer (left side of each mouse) grew significantly larger than Ad-HK infected tumors (right side of each mouse).

gulation of Dicer might impact tumor cells in vivo, we assessed the growth of subcutaneous tumor xenograft of MCF-7 following infection with Ad-Dicer. At the onset of infection, the mean tumor volumes of $35.66 \pm 26.49$ and $38.27 \pm 15.29 \mathrm{~mm}^{3}$, respectively, for the Ad-Dicer and Ad-HK infected groups, were not statistically significant from one another. During the first week of the observation period, the tumors in both the Ad-HK groups and Ad-Dicer groups grew slowly and there was no observable difference in tumor size. Starting at day 8 after the initial treatment, however, the tumors in the Ad-Dicer-infected group grew significantly more rapid than those infected with Ad-HK (Fig. 5). The mean volumes of tumors were $1023.50 \pm 266.68$ and $783.38 \pm 299.27 \mathrm{~mm}^{3} 20$ days following infection with AdDicer or Ad-HK, respectively $(\mathrm{p}<0.01)$. Following the 20 -day observation period, mice were sacrificed and tumors were excised and paraffin-embedded for immunohistopathological examination. Similar to the results obtained in vitro, the tumor specimens infected with Ad-Dicer resulted in downregulation of Dicer expression, as well as displayed enhanced expression of p-AKT, MMP2/9, cyclin A and PCNA. Each of these proteins were significantly upregulated compared to Ad-HK treated groups (Fig. 6). In sum, infection of tumor cells with Ad-Dicer resulted in increased proliferation both in vitro and in vivo, and resulted in a similar enhanced 


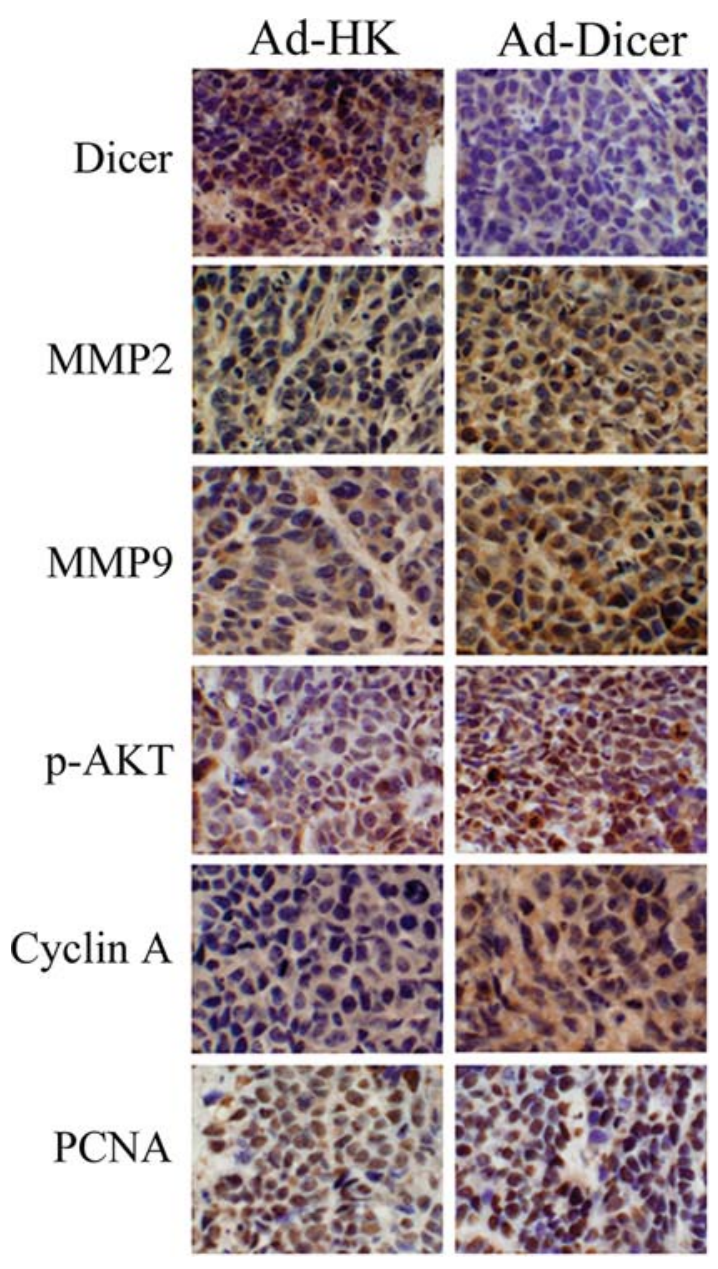

Figure 6. The expression of Dicer, p-AKT, MMP2/9, cyclin A and PCNA in vivo were detected by immunohistochemistry. The results were consistent with that observed in vitro. Dicer protein was effectively inhibited by rAdDicer treatment, resulting in upregulation of p-AKT, MMP2/9, cyclin A and PCNA in rAd-Dicer infected tumors.

protein expression profile of $\mathrm{p}-\mathrm{AKT}, \mathrm{MMP} 2 / 9$, cyclin A and PCNA.

\section{Discussion}

Here, we identify a relationship between downregulation of Dicer and the presumed impairment of miRNA processing with the transformation of tumor cells. We speculate that the impairment of miRNAs processing by Dicer inhibition promoted the proliferative and the invasive ability of tumor cells. Recent studies have shown a general decrease in miRNAs in tumors deriving from multiple tissues. Lu et al analyzed 217 mammalian miRNAs from 334 cancer samples, and revealed a global reduction of mature miRNA levels in both human and mouse cancers (2). Additional studies revealed that reduced expression of Dicer was observed in a significant fraction of lung cancers, and associated with shorter post-operative survival (3), and that conditional deletion of Dicer enhanced tumor development in a K-Ras-induced mouse model of lung cancer. These findings imply that impaired miRNAs processing may be involved in tumorigenesis. Our study corroborates these findings, demonstrating that downregulation of a key component in the miRNA processing machinery promotes the malignant transformation of tumor cells. Our findings are also consistent with the observation that miRNA processing-impaired cells generate tumors with accelerated kinetics in animals (17). Taken together, these data indicate that global miRNA loss promotes the malignant phenotype of tumor cells and facilitates tumorigenesis.

Although the knockdown of Dicer has been associated with enhanced tumor proliferation and invasion, the mechanism of this event remains elusive. In our study we began investigation of several lead proteins that may impact cell proliferation and invasive ability. The PI3K/AKT pathway is constitutively activated in a wide range of tumor types, and triggers a cascade of responses ranging from cell growth and proliferation to survival and motility (21). Here we identify that impairment of miRNA results in the enhanced phosphorylation of AKT. We demonstrate in both in vitro and in vivo systems, knockdown of Dicer expression significantly increased expression of p-AKT compared to mock or control adenovirus infections. Based upon these findings, we speculate that the enhanced tumor progression that we observed following Dicer knockdown may be attributed to enhanced activation of the AKT signaling pathway.

A primary biological consequence of AKT activation is activation of cellular proliferation via regulation of the cellcycle machinery. Indeed, our investigation identified a significant increase in cyclin A expression after downregulation of Dicer. Cyclin A, a primary member of the cyclin family, is a component of the machinery required for progression through $\mathrm{S}$ phase $(22,23)$. Constitutive expression of cyclin A has been associated with tumorigenesis in multiple studies $(24,25)$. Increased expression of this protein was consistent with the outcome of cell cycle analysis, which showed that the cell cycle was promoted to $\mathrm{S}$ phase following infection, suggesting that a link between impairment of miRNA processing, AKT phosphorylation and activation of the cell cycle machinery via cyclin A may exist. Furthermore, we observed a significant increase in the expression of proliferating cell nuclear antigen (PCNA), a DNA clamp protein required for processing of the leading strand during DNA replication following silencing of Dicer. Both cyclin A and PCNA were similarly upregulated in tumor xenografts infected with the Dicer-silencing adenovirus. While speculative, these findings warrant additional study.

A second major biological consequence of AKT phosphorylation is the activation of tumor cell invasion properties via induction of Matrix metalloproteinases (MMPs). The MMPs are a family of enzymes responsible for the proteolytic processing of extracellular matrix structural proteins, an essential step in the invasion process (26). Many investigations have demonstrated that MMP-2 and MMP-9 secretion is elevated in several types of human cancers and their elevated expression has been associated with poor prognosis (27). Previous studies also have shown that the expression of both MMP2 and MMP-9 were modulated by activation of AKT and MAPK $(28,29)$. We became interested in the expression profile of the MMP proteins as we observed increased invasive ability of Ad-Dicer-infected tumor cells to migrate in the transwell assay. Further, as we observed an increase in the phosphorylation state of AKT, we were not surprised to have observed a 
similar increase in the expression of both MMP-2 and MMP-9 proteins. We validated these findings by demonstrating a similar increase in expression profile both in vitro and in vivo. Based upon these findings, we hypothesize that impaired miRNA processing enhances the metastasic potential of a tumor by p-AKT-mediated stimulation of MMP-2 and MMP-9. As such these findings may have significant clinical value.

In summary, our study contributes important new findings for the role of Dicer-mediated miRNA processing in tumor cell proliferation and invasion. We investigated the phenotypes of three tumor cell lines after global impairment of miRNA processing by adenoviral knockdown of the expression of Dicer. Our studies identified that global downregulation of miRNA maturation led to an enhanced proliferative and invasive ability in tumor cell lines. While we speculate that the mechanism involved in the cell transformation was a result of phosphorylation of AKT, leading to activation of the cell cycle machinery and MMPs, further study of this signaling pathway is warranted. Multiple questions remain, such as identification of the key miRNAs influenced by Dicer downregulation that activate AKT, and whether p-AKT directly activates the cell cycle machinery and MMPs or if this phenomenon is attributed to another, yet undefined consequence of Dicer downregulation.

\section{Acknowledgements}

This work was supported by China National Natural Scientific Found (30971136, 30772231), Program for New Century Excellent Talents in University (NCET-07-0615) and Natural Science Foundation of Tianjin Municipal Science and Technology Commission (09JZCD17600) to C.S. Kang.

\section{References}

1. Ouellet DL, Perron MP, Gobeil LA, Plante P and Provost P: MicroRNAs in gene regulation: when the smallest governs it all J Biomed Biotech 2006: 1-20, 2006.

2. Lu J, Getz G, Miska EA, et al: MicroRNA expression profiles classify human cancers. Nature 435: 834-838, 2005.

3. Karube Y, Tanaka H, Osada H, et al: Reduced expression of Dicer associated with poor prognosis in lung cancer patients. Cancer Sci 96: 111-115, 2005.

4. Michael MZ, O'Connor SM, Van Holst PG, Yong GP and James RJ: Reduced accumulation of specific microRNAs in colorectal neoplasia. Mol Cancer Res 1: 882-891, 2003.

5. Calin GA, Dumitru CD, Shimizu M, et al: Frequent deletions and down-regulation of micro-RNA genes miR-15 and miR-16 at 13 q14 in chronic lymphocytic leukemia. Proc Natl Acad Sci USA 99: 15524-15529, 2002.

6. Takamizawa J, Konishi H, Yanagisawa K, et al: Reduced expression of the let-7 microRNA in human lung cancers in association with shortened postoperative survival. Cancer Res 64: 3753-3756, 2004.

7. He L, Thomson JM, Hemann MT, et al: A microRNA polycistron as a potential human oncogene. Nature 43: 828-833, 2005.

8. Hayashita Y, Osada H, Tatematsu Y, et al: A polycistronic microRNA cluster, miR-17-92, is overexpressed in human lung cancers and enhances cell proliferation. Cancer Res 65: 9628-9632, 2005.
9. Chan JA, Krichevsky AM and Kosik KS: MicroRNA-21 is an anti-apoptotic factor in human glioblastoma cells. Cancer Res 65: 6029-6033, 2005.

10. Carmell MA and Hannon GJ: RNase III enzymes and the initiation of gene silencing. Nat Struct Mol Biol 11: 214-218, 2004.

11. Yi R, Qin Y, Macara IG and Cullen BR: Exportin-5 mediates the nuclear export of pre-microRNAs and short hairpin RNAs. Genes Dev 17: 3011-3016, 2003.

12. Grishok A, Pasquinelli AE, Conte D, et al: Genes and mechanisms related to RNA interference regulate expression of the small temporal RNAs that control C. elegans developmental timing. Cell 106: 23-34, 2001.

13. Hutvagner G, McLachlan J, Pasquinelli AE, Balint E, Tuschl T and Zamore PD: A cellular function for the RNA-interference enzyme Dicer in the maturation of the let-7 small temporal RNA. Science 293: 834-838, 2001.

14. Kanellopoulou C, Muljo SA, Kung AL, et al: Dicer-deficient mouse embryonic stem cells are defective in differentiation and centromeric silencing. Genes Dev 19: 489-501, 2005.

15. Harfe BD, McManus MT, Mansfield JH, Hornstein E and Tabin CJ: The RNase III enzyme Dicer is required for morphogenesis but not patterning of the vertebrate limb. Proc Natl Acad Sci USA 102: 10898-10903, 2005.

16. Muljo, SA, Ansel KM, Kanellopoulou C, Livingston DM, Rao A and Aberrant KR: T cell differentiation in the absence of Dicer. J Exp Med 202: 261-269, 2005.

17. Kumar MS, Lu J, Mercer KL, Golub TR and Jacks T: Impaired microRNA processing enhances cellular transformation and tumorigenesis. Nat Genet 39: 673-677, 2007.

18. Chiosea S, Jelezcova E, Chandran U, Acquafondata M, McHale T, Soboland RW and Dhir R: Up-regulation of Dicer, a component of the microRNA machinery, in prostate adenocarcinoma. Am J Pathol 169: 1812-1820, 2006.

19. Pu PY, Kang CS, Zhang ZY, Liu XZ and Jiang H: Downregulation of PIK3CB by siRNA suppresses malignant glioma cell growth in vitro and in vivo. Technol Cancer Res Treat 5: 271-280, 2006.

20. Doi N, Zenno S, Ueda R, Ohki-Hamazaki H, Ui-Tei K and Saigo K: Short-interfering-RNA-mediated gene silencing in mammalian cells requires Dicer and eIF2C translation initiation factors. Curr Bio 13: 41-46, 2003.

21. Vivanco I and CL Sawyers: The phosphatidylinositol 3-kinase AKT pathway in human cancer. Nat Rev Cancer 2: 489-501, 2002.

22. Krek W, Ewen ME, Shirodkar S, Arany Z, Kaelin WG and Livingston DM: Negative regulation of the growth-promoting transcription factor E2F-1 by a stably bound cyclin A-dependent protein kinase. Cell 78: 161-172, 1994.

23. Lees E, Faha B, Dulic V, Herd SI and Harlow E: Cyclin E/cdk2 and cyclin A/cdk2 kinases associate with p107 and E2F in a temporally distinct manner. Genes Dev 6: 1874-1885, 1992.

24. Wang J, Chenivesse X, Henglein B and Brechot C: Hepatitis B virus integration in a cyclin A gene in a hepatocellular carcinoma. Nature 343: 555-557, 1990.

25. Barlat I, Fesquet D, Strausfeld U, Brechot C, Henglein B, Vie A and Blanchard JM: Loss of the G 1-S control of cyclin A expression during tumoral progression of Chinese hamster lung fibroblasts. Cell Growth Differ 4: 105-113, 1993.

26. Karagiannis ED and Popel AS: Distinct modes of collagen type I proteolysis by matrix metalloproteinase (MMP) 2 and membrane type I MMP during the migration of a tip endothelial cell: Insights from a computational model. J Theor Biol 238: 124-145, 2005.

27. Stetler-Stevenson WG: The role of matrix metalloproteinases in tumor invasion, metastasis and angiogenesis. Surg Oncol Clin N Am 10: 383-392, 2001.

28. Suzuki A, Lu J, Kusakai G, Kishimoto A, Ogura T and Esumi H: ARK5 is a tumor invasion-associated factor downstream of Akt signaling. Mol Cell Biol 24: 3526-3535, 2004.

29. Thant AA, Nawa A, Kikkawa F, et al: Fibronectin activates matrix metalloproteinase-9 secretion via the MEK1-MAPK and the PI3K-Akt pathways in ovarian cancer cells. Clin Exp Metastasis 18: 423-428, 2000. 